ISSN: $2637-4692$

\title{
Signs of Tooth Eruption in Infants
}

\author{
Karimi* \\ Sepideh Dental Clinic, Department of Pediatric Dentistry, Iran
}

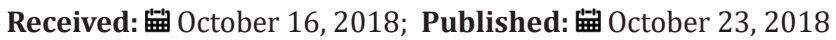

*Corresponding author: Karimi, Sepideh Dental Clinic, Department of Pediatric Dentistry, Iran

\begin{abstract}
Teething is one of the challenges of medical science in Infancy period. Many studies show that teeth eruptions might have a lot of symptoms, including bad mood, skin rashes on the cheeks and around the mouth, increasing saliva secretion, swollen gums, Sucking a finger and etc. These signs and symptoms are mild in some infants, and in others may be severe; it is difficult for parents to tolerate these conditions. The peak of the severity of teething-related symptoms is when the dental buds have grown sufficiently in the gum and try to exit from the swollen gingiva.
\end{abstract}

Keywords: Teething; Saliva Secretion; Bad Mood; Skin Rash; Swollen Gum; Dental Buds

\section{Introduction}

As important as it is, tooth eruption is a matter of difficulty for both parents and children. Note that maintaining the health of teeth and gums in the child is the basis of the health of his teeth and gums for whole life. Teeth eruption is one of the most important stages of the growth, and through this stage, the baby will be able to have chewable foods. Commonly, there is a timetable for each primary tooth to erupt in the mouth. Signs of infant's teeth eruption include disturbances in sleep and night-time wakening, mouth-watering, redness of cheeks, chewing fingers and objects, swollen gums, child malaise, bad temper, and the appearance of tooth buds [1-3]. In this article, we refer to any of these cases.

\section{Schedule the Growth of Different Teeth in the Baby}

Generally, new teeth grow in pairs. First, we see the eruption of the two lower primary central incisors teeth, and about a month later, the two upper primary central incisors teeth would erupt. Of course, in some cases, the four lower primary anterior teeth might erupt at first, and then we see the eruption of the upper primary anterior teeth or vice versa. In general, the eruptions of the primary teeth are scheduled to be as follows: [4]

a) At 6 months of age: lower primary central incisors teeth

b) At 8 months of age: upper primary central incisors teeth

c) At 10 months of age: upper and lower primary lateral teeth

d) At 14 months of age: first primary molars teeth

e) At 18 months of age: primary canines' teeth

f) At age 2: second primary molars.

\section{Signs and Symptoms}

Given that the primary teeth are much smaller when they come out of the gum, and the process of tooth eruption is different in children, the symptoms that can usually be expected are:

a) Distracted Dreams: In some children, teething might be a painful process that can awake the baby at night. Therefore, if the baby suddenly wakes up at night, it may be due to her teeth. At this time, the best thing that mothers can do is taking her to the cradle and calm down.

b) Excessive Mouthwatering: Increasing saliva secretion can be one of the symptoms of the eruption of a new tooth. This is, of course, one of the natural stages of the baby's teeth growth, so too much water in the mouth is not always a sign of baby's teething. There is no way to detect the association between excessive saliva secretion and the eruption of new teeth. In a baby who is teething, his chin is often wet. Excessive saliva can cause injury and irritation of the baby's face, so mothers should dry the baby's face and mouth with a soft, delicate cloth. To protect the skin of the baby's face, they can consult with their physician.

c) Swollen Gums: In some cases, before germinating of the tooth, the growth site in the gingiva of the infant becomes red, swollen and bruised. Sometimes, the rise of a tooth below the gum causes a bulge so that if parents can persuade her child to open the mouth enough, they can see the whitish shadow of the tooth under the swollen gingiva.

d) Chewing Things: Children like to take everything in their mouths, but if the baby too much does that, she may be teething. 
The pressure that unerupted teeth from below have on the gums, make the pain to be relieved by applying pressure from the opposite direction. Hence, most infants who are teething are willing to bite on different objects. Mothers should try to give them a teether rings to make them a little quiet. Of course, chewing on different things can also be the innate response of the baby to the strange sense of the mouth.

e) Sucking a Finger: In addition to biting on things during teething, it may be accompanied by sucking a finger. The baby can suck and bites for a few hours during the day. By doing this, he also tries to eliminate the pruritus of the gums. The joy of this action helps the baby to eliminate the pain and pressure of the teeth. Therefore, a mother may find that her baby calms down by chewing his fingers. She should try to keep her baby's hands clean so no microorganisms can enter the baby's mouth.

f) Change in Eating Habits: Wounds and gum's swelling can make sucking painful for the baby. If the baby is hungry but runs away from feeding by mother's breast or bottle, he may be teething. In this case, babies who eat solid foods tend to breastfeed or feed on the bottle, because the spoon annoys their inflamed gums. Some other babies also do quite the opposite of doing this, that is, they eat more because the bilateral pressure gives them a good feeling. On the other side, babies who still feed on the breast or bottle may eagerly start feeding at the beginning but quickly refrain from eating, because the sucking action puts very uncomfortable pressure on the gums and ear canals.

g) Baby's Temper Tantrum: Primary tooth eruption (the outward movement of the tooth in the bone and gum) is usually done in a manner so that this action takes place more often overnight compared to the daytime, consequently, the baby will be more restless at night. The pain of teething can cause her sensitivity and irritability, and make him constantly cry.

h) Acne: In some children, teeth eruption may be accompanied with symptoms such as acne. Of course, this symptom is not definitive like fever, and it may have other causes. Skin hypersensitivities are more likely in children due to delicateness and vulnerability of their skin, and occasionally these acnes are signs of gastrointestinal symptoms.

i) Pulling the Ears: Holding, gripping or even pulling the ears, although sometimes indicative of ear infections, it can also be a sign of tooth eruption; in this situation, the pain that occurs in the jaw could be a transferred pain to the ears.

j) Cough: In some cases, when children are teething, they start coughing at that period of time.

\section{Two Misconceptions about Signs of Teething}

Many still believe that a child can have a fever during teething 5]] (even healthcare providers!), but on the contrary, some believe the temperatures above 102 degrees Fahrenheit are not related to teething [6]. If fever exists in this period, it is a mere accident and must be caused by other factors [7]. However, if we compare the temperature of the baby's body who is teething with a child who does not, his body temperature may be slightly high, but this increase is not important enough to be called fever. Hence, why do many babies with teeth eruption have a fever? What is the explanation for that? Another misconception is that teething causing diarrhea $[8,9]$. Severe diarrhea or constipation is not associated with teeth eruption, and these two events should normally not be accompanied with each other. Although fever and diarrhea are not so much related to the teething, interestingly these signs are seen in some children with the onset of teeth eruption. The concurrency of diarrhea, fever and tooth eruption has two main causes: first, around the age of 4-6 months, when the teeth are emerging, the child's immune system gradually become independent of the mother reduces the body's resistance, and therefore this causes symptoms such as fever and diarrhea. Secondly, at this time, children take everything in their mouths to relieve the itching of the gums and discomfort that they develop during teething, but these objects may be contaminated to microbes [10], hence, there is the expectation of diarrhea in infants. Consequently, the emerging of teeth itself does not cause severe diarrhea. On the other hand, at this time, the safety of the immune system from the mother to the infant will diminish, [11] and the child's body must build the safety components. Therefore, the reduction of maternal immunity which is transferred from the mother to the child can be a reason for fever or infection, [11] which is only a concurrency with teething; and the tooth eruption alone, do not cause this symptom.

\section{Final Words}

To summarize the article, the eruption of the teeth is a natural occurrence that occurs without acute and severe problems. It is a physiological phenomenon that will be associated with the other physiological phenomena such as increased saliva in the child, gums swelling, biting on any objects, disturbance in night sleepless, changes in eating habits, and so on.,. The tooth eruption does not pose a problem for the child. Some people mistakenly think that when the baby wants to have a new tooth, he is prone to having a fever, severe diarrhea or even constipation. The growth of teeth will never be accompanied by high fever. The teeth eruptions are different in newborns, but in general, most of them have first teeth at six months of age. Noteworthy that some of the symptoms of teething are similar to those of some diseases, which should be contacted with a pediatric pediatrician in the event of worsening of the symptoms.

\section{References}

1. Markman L (2009) Teething: facts and fiction. Paediatr. Rev 30(8): e59-e64.

2. Tsang AKL (2010) Teething, teething pain and teething remedies. International Dentistry SA 12(5): 48-61. 
3. Eisenstadt M, Malkiel S, Pollak U (2017) It's Alright, Ma (I'm only Teething...) dispelling the Myth from the Teeth. Acad J Ped Neonatol 3(4): $1-4$

4. Yeung $\mathrm{CY}$, Chu $\mathrm{CH}$ (2014) A review of the eruption of primary teeth. OA Dentistry 2(1): 7.

5. Jaber L, Cohen IJ, Mor A (1992) Fever associated with teething. Arch Dis Child 67: 233-234.

6. Michael L Macknin, Marion Piedmonte, Jonathan Jacobs, Christine Skibinski (2000) Symptoms Associated with Infant Teething: A Prospective Study. Pediatrics 105(4).
7. Vincent Iannelli MD (2018) How to Soothe a Teething Baby's Symptoms.

8. Hassanali J, Amwayi P, Muriithi A (1995) Removal of deciduous canine tooth buds in Kenyan rural Maasai. East Afr Med J 72: 207-209.

9. Wake M, Hesketh K, Allen M (1999) Parent's beliefs about infant teething: A survey of Australian parents. J. Paediatr child health 35(5): 446-449.

10. Foster TD, Hamilton MC (1969) Occlusion in the primary dentition. Study of children at 2 and one-half to 3 years of age. Br Dent J 126(2): 76-79.

11. Seward MH (1971) Local disturbances attributed to eruption of the human primary dentition, a survey. Br Dent J130(2): 72-77.
(C) This work is licensed under Creative

To Submit Your Article Click Here: Submit Article

DOI: 10.32474/MADOHC.2018.03.000162

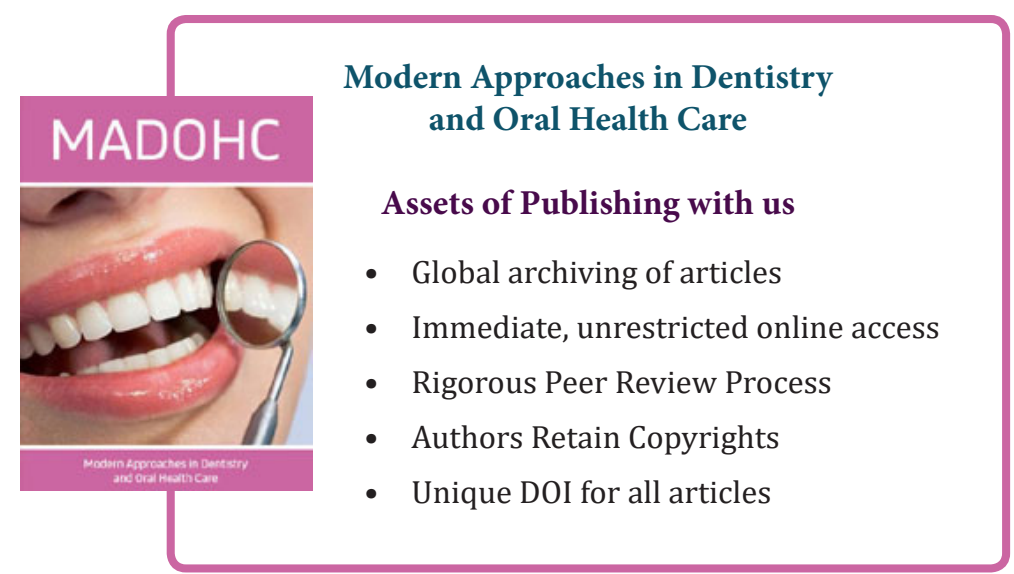

